\section{(A) Check for updates}

Cite this: Analyst, 2020, 145, 5878

\title{
Detection and time-tracking activation of a photosensitiser on live single colorectal cancer cells using Raman spectroscopy $\dagger$
}

\author{
Julia Gala de Pablo, (DD ${ }^{\text {a,b }}$ David R. Chisholm, ${ }^{c, d}$ Carrie A. Ambler, ${ }^{c, e}$ \\ Sally A. Peyman, ${ }^{a, f}$ Andrew Whiting (DD ${ }^{c, d}$ and Stephen D. Evans ${ }^{* a, f}$
}

\begin{abstract}
Raman spectroscopy has been used to observe uptake, metabolism and response of single-cells to drugs. Photodynamic therapy is based on the use of light, a photosensitiser and oxygen to destroy tumour tissue. Here, we used single-cell Raman spectroscopy to study the uptake and intracellular degradation of a novel photosensitiser with a diphenylacetylene structure, DC473, in live single-cells from colorectal adenocarcinoma cell lines SW480, HT29 and SW620. DC473 was seen to predominantly accumulate in lipid droplets, showing higher accumulation in HT29 and SW620 cells than in SW480 cells, with a broader DC473 peak shifted to higher wavenumbers. DC473 activation and effects were tracked on live singlecells for 5 minutes. Upon exposure to UV light, the DC473 signal intensity dropped, with remaining DC473 shifting towards higher wavenumbers and widening, with a lifetime of approximately 50 seconds. Morphologically, SW480 and SW620 cells showed changes upon photodynamic therapy, whereas HT29 cells showed no changes. Morphological changes correlated with higher remaining DC473 signal after UV exposure. Our research suggests that DC473 forms aggregates within the cells that disaggregate following activation, showing the potential of Raman spectroscopy for the study of time-dependent single-cell pharmacodynamics.
\end{abstract}

Received 21st May 2020

Accepted 6th July 2020

DOI: 10.1039/d0an01023e

rsc.li/analyst related to the cell response. ${ }^{9-11}$ Raman has been extensively applied on pharmacological characterization. ${ }^{12}$

The spontaneous Raman signal is weak, making the technique inherently slow. ${ }^{4}$ Additionally, drug peaks often appear in the cell fingerprint region, merging with bands from components such as proteins, lipids and nucleic acids. ${ }^{13}$ For tracking a drug within a cell, alternative approaches can be used, such as Resonance Raman scattering (RRS), the use of isotopologues such as deuterium labelling ${ }^{14,15}$ or biorthogonal Raman tagging such as through alkyne incorporation. ${ }^{13,16-18}$ RRS has been employed to study cytochrome C at $532 \mathrm{~nm},{ }^{19,20}$ where a drop in signal intensity, due to oxidation, has been observed at early stages of apoptosis. ${ }^{19}$ Biorthogonal Raman tagging uses molecules that exhibit vibrational bands in the Raman silent region of cells (1800-2800 $\mathrm{cm}^{-1}$ ) such as alkynes, enabling more accurate detection of compounds in single cells. ${ }^{16-18,21-26}$ Alternatively, non-linear RS, such as Coherent anti-Stokes RS (CARS) ${ }^{27}$ or Stimulated RS (SRS) ${ }^{28}$ can be used though they require a more complicated setup with multiple laser beams making their translation to medical and biological applications challenging. ${ }^{29}$

RS has been used to observe both uptake, metabolism and response of single cells to drug treatment, ${ }^{12}$ and is often carried out post fixation of the cells, thereby marking an end- 
point to the experiment. For example, Raman microspectroscopic studies were done on the response of cancer cells to tyrosine kinase inhibitors containing $-\mathrm{C} \equiv \mathrm{C}-$ or $-\mathrm{C} \equiv \mathrm{N}$ moieties in lung cancer, ${ }^{1}$ colorectal cancer $^{30-32}$ and leukaemia, ${ }^{33}$ and for detecting drug metabolism within the cell. ${ }^{34,35}$ Yamakoshi et al. used RS to image the different chemical forms of a mitochondrial uncoupler with a nitrile Raman tag on live HeLa cells. ${ }^{18}$ Spectroscopic studies have also been used to detect cisplatin binding to DNA in the nucleus ${ }^{36}$ cell membrane and cytoplasm $^{37}$ of lung adenocarcinoma cells. Doxorubicin accumulation and the cell's response at different incubation times was measured on fixed cells using RS. ${ }^{38,39}$ Internalization of biodegradable biocarrier systems in single cells has been extensively studied with Raman microspectroscopy, ${ }^{12,40}$ with multiple studies on poly(lactideco-glycolide) beads. ${ }^{41-45}$ Microspectroscopy studies give insights into the responses to the drugs of interest, however, mapping is time consuming and low throughput, usually in the tens of cells. In addition, using fixed cells precludes the ability to perform time-dependent studies on the same singlecell.

Colorectal cancer (CRC) is the third most common cancer worldwide and second in mortality, with over 1.8 million new cases diagnosed in 2018, and more than 850000 deaths. ${ }^{46}$ Approximately half of the patients develop metastasis to the liver ${ }^{47}$ and resistance to standard chemotherapy. ${ }^{48}$ Novel therapies for the treatment of CRC are, therefore, needed. Photodynamic Therapy (PDT) is based on the use of light, a photosensitizer (PS) and oxygen to destroy tumour tissue through oxidative damage, vascular shutdown and activation of immune response against cancer cells. ${ }^{49}$ DC473 is a low molecular weight photosensitiser that can be activated by excitation with UV-A, violet or corresponding near-infrared twophoton wavelengths. DC473 exhibits strong solvatochromic fluorescence and possesses two alkyne groups. ${ }^{25,50,51}$ Previous in vitro studies have reported epithelial cell death, after UV-A irradiation following incubation with DC473, due to the production of reactive oxygen species. ${ }^{51}$

One of the first reports of Raman detection of photosensitisers within cells was the publication by Freeman et al. in 1997, detecting zinc phthalocyanines in a fixed human endothelial hybridoma cell line. ${ }^{52}$ Abramczyk et al. used Raman microspectroscopy and observed preferential accumulation of aluminium phthalocyanine cancerous breast tissue compared to non-cancerous tissue. ${ }^{53,54}$ Brozek-Pluska et al. used RS to provide a map of lipids and proteins and fluorescence to detect hematoporphyrin and phthalocyanine in healthy and cancerous breast tissue, showing higher accumulation in the latter. ${ }^{55,56}$ In vivo, Bhattacharjee et al. performed RS measurements in breast cancer model rats showing PS accumulation and PDT effects of chlorin photodithazine. ${ }^{57}$ An alternative approach is to use Surface-Enhanced RS (SERS) to increase the limit of detection of the PS, often done by including metal nanoparticles in theranostic constructs with the PS. ${ }^{58-64}$ Fales et al., used targeted gold nanostar constructs loaded with PPIX, showing detection and targeting in single cells, ${ }^{65,66}$ and
Farhadi et al. coupled palladium porphyrin with plasmonic nanoparticles to allow SERS detection of the PS, using the same wavelength to excite Raman scattering and to stimulate PDT. $^{67}$ To our knowledge, there have been no reports in the detection of PS accumulation in live single cells using RS, or in the single-cell pharmacodynamics of the PS upon activation.

In this manuscript, we study both the accumulation and the photoactivation of a novel photosensitiser, DC473, in live single-cells from cell lines representing different stages of colorectal cancer. For our model we used an adenocarcinoma model system comprised by the cell lines SW480, HT29 and SW620, representing Duke's stage B primary, Duke's stage C primary and Duke's stage C secondary cell lines, respectively. ${ }^{20}$

\section{Methods}

\section{Cell preparation}

Cells were cultured and measured as in our previous work. ${ }^{20}$ The SW480, HT29 and SW620 cell lines were cultured in Dulbecco's Modified Eagle Medium (DMEM/F-12, Gibco) supplemented with $10 \%$ fetal bovine serum (Sigma), $2 \mathrm{mM}$ Glutamax (Thermo Fisher Scientific) and penicillin 100 units per $\mathrm{mL}$ streptomycin $100 \mu \mathrm{g} \mathrm{mL}^{-1}$ (Sigma). All experiments were done with passage numbers below 50 .

\section{DC473 incubation}

Cells were seeded 2 days in advance of the experiment in 6 well plates at a concentration of $3.5 \times 10^{5}$ cells per well. Before Raman measurements, media was changed and $4 \mu \mathrm{M}$ of DC473 in a $0.4 \%$ DMSO/media solution was added to the cells and incubated for $4 \mathrm{~h}$, whereas the control cells received an equivalent concentation of DMSO $(0.4 \%)$. Prior to the experiment, cells were washed with Dulbecco's phosphate-buffered saline (DPBS) and gently retrieved from 6-well plates by incubating with TrypLe (Thermo Fisher Scientific) for $5 \mathrm{~min}$, followed by centrifugation and two washes in DPSB (100 gs $1 \mathrm{~min}$ ) and re-suspension in cell dissociation buffer (Thermo Fisher). When pipetted into the setup, cells sedimented onto the coverslip and showed no visible Brownian motion, remaining in a spherical shape.

\section{Raman spectroscopy}

Quartz slides (UQG Optics, $75 \times 25 \times 1 \mathrm{~mm}$ ) and coverslips $(25.4 \times 25.4 \times 0.15-0.25 \mathrm{~mm}$ Alfa Aesar $)$ were sonicated with acetone (VWR Chemicals), 2-5\% Decon 90 (VWR Chemicals) and rinsed with MilliQ. All organics were removed by incubation for 20 minutes in Piranha solution $\left(\mathrm{H}_{2} \mathrm{O}_{2} 30 \%\right.$ (Thermo Fisher) and $\mathrm{H}_{2} \mathrm{SO}_{4}>95 \%$ (Thermo Fisher) in a $3: 7$ proportion). Spacers were prepared using a $50 \mu \mathrm{m}$ polyethylene terephthalate film (Goodfellow, UK). A nitrocellulose-based solution was used to bond the coverslip to the slide. The cell solution was pipetted into this chamber immediately before measuring. All experiments were done at room temperature and samples were measured for $1 \mathrm{~h}$. 
The Raman system used was an inVia Raman confocal inverted microscope (Renishaw) integrated with a Leica DMi8/ SP8 laser scanning confocal microscope system, with a DPSS Diode $532 \mathrm{~nm}$ laser (laser power of $22 \mathrm{~mW}$ on the sample) and a $785 \mathrm{~nm}$ laser (laser power of $45 \mathrm{~mW}$ at the sample), with gratings of $1800 \mathrm{l} \mathrm{mm}^{-1}$ and $1200 \mathrm{l} \mathrm{mm}^{-1}$ respectively. Light was collected using either a Newton EMCCD Sensor (DU970P, Andor, $1600 \times 200 \mathrm{px}$ ) for the visible excitation laser or a nearinfrared enhanced CCD array detector $(1024 \times 256$ pixels, Renishaw). Before each experiment a spectrum of a silicon sample was collected using the respective objective, grating and detectors used and the microscope was calibrated to the peak position $\left(520.5 \mathrm{~cm}^{-1}\right)$.

The cell spectra were obtained using a $100 \times$ oil objective (HC PL APO CS2 FWD $0.13 \mathrm{~mm}$ NA 1.4) and a slit size of either $20 \mu \mathrm{m}$ (for DC473 accumulation experiments and DC473 off cell activation experiment) or $65 \mu \mathrm{m}$ (for DC473 PDT experiments). This objective gave a $10.8 \mu \mathrm{m}$ and $25.7 \mu \mathrm{m}$ Full-Width Half Maximum axial confocality when tracking the changes of Raman intensity of the $520.5 \mathrm{~cm}^{-1}$ with the distance to a silicon sample for each of the slit openings. The laser spot was defocused by $20 \%$ using a beam expander, generating a laser spot of approximately $18 \mu \mathrm{m}$ diameter, and obtaining the average signal of the cell. Cell spectra for DC473 accumulation were obtained using a step configuration with $1 \mathrm{~s}$ exposure time and 5 accumulations in two different windows (300-1800 $\mathrm{cm}^{-1}$ and 1800-3200 $\mathrm{cm}^{-1}$ ) which gave a total exposure time of $10 \mathrm{~s}$ per cell. For the DC473 PDT experiments, the $300-1800 \mathrm{~cm}^{-1}$ region was prioritized to increase the spectral quality and time resolution. The data from multiple experiments were combined for this paper without omitting any outliers, with 135 cells incubated with DC473 and 134-135 cells as control for each cell line. Five background spectra from cell-free regions of the sample measured at the same $Z$-position as the cells were obtained for each experiment.

\section{Photodynamic activation of DC473}

DC473 off-cell activation was done using a $100 \mu \mathrm{M}$ DC473 solution in DMSO, using a $100 \times$ oil immersion objective and 340-380 nm UV light with a light intensity of $131 \mathrm{~mW} \mathrm{~cm}^{-2}$ and a total dose of $5.2 \mathrm{~J} \mathrm{~cm}^{-2}$. Acquisition was obtained using the $785 \mathrm{~nm}$ excitation before and immediately after activation $(1.13 \mathrm{~mJ})$. For tracking the activation of DC473 on live single cells, before cell treatment, a Raman spectrum was acquired setting the system to acquire spectra every $20 \mathrm{~s}$. Then, the PS was activated using $3 \mathrm{~s}$ of UV light, for a total dose of $711 \mathrm{~mJ}$ $\mathrm{cm}^{-2}$. The following acquired spectra tracked the changes in the cell following the PS activation. Two controls were made, with cells incubated with DMSO exposed to the Raman acquisition light and cells incubated with DMSO exposed to both the Raman acquisition light and the UV light, to understand the phototoxicity due to these light sources in the absence of the PS. Typically, 10-11 cells were measured per group, acquiring 15 spectra per cell, with a total of around 30-35 cells per cell line.

\section{Pre-processing of the spectra}

The spectra obtained were cosmic ray filtered (WiRE® software) and exported as text files for further analysis using Matlab's Statistics and Machine Learning Toolbox (MathWorks). The Matlab functions used are indicated by italics. The silicon peak of a calibration sample was used to calibrate the wavenumber axis of each spectrum and the spectra were translated vertically, such that the minimum intensity was zero. For each cell spectrum, the average background spectrum of the sample was multiplied by an adjustment factor before being subtracted from the cell spectrum. The spectra were truncated to only consider the regions between $730 \mathrm{~cm}^{-1}$ and $3100 \mathrm{~cm}^{-1}$. The spectra were baseline corrected using the algorithm developed by Koch et al. (2016). ${ }^{68}$ The regions of the spectra between $1750 \mathrm{~cm}^{-1}$ and $2800 \mathrm{~cm}^{-1}$ were not considered for subsequent analysis. In order to normalize to the protein content, for comparison with biochemical literature data, each spectrum was normalized such that the Amide I peak was unity. For the PDT experiments, where only the fingerprint region was acquired, similar preprocessing was followed.

\section{Statistical analysis and classification}

Statistical errors. Unless stated otherwise, all values are expressed \pm the standard error (SE) calculated as $\sigma / \sqrt{ } N$, where $\sigma$ is the standard deviation and $N$ the sample size. PCA. The edited data was standardized using Standard Normal Variate. PCA was used to identify the main sources of variability in the datasets. The function used was pca. Significance. Significance was calculated by comparing the single cell observations using unequal variances $t$-tests. GLS fitting. GLS fitting was done based on the description from Jain et al., using Matlab. ${ }^{69}$ For the $2195 \mathrm{~cm}^{-1}$ band, the peak was allowed a variation in the position of $\pm 10 \mathrm{~cm}^{-1}$ and a width of $20 \pm 10 \mathrm{~cm}^{-1}$. For the $1597 \mathrm{~cm}^{-1}$ band, the fitting was more challenging due to overlap from the adjacent Amide I and cytochrome C bands. Three bands were fitted for the Amide I; namely: $\alpha$-helix $\left(1648 \mathrm{~cm}^{-1}\right), \beta$-sheet $\left(1667 \mathrm{~cm}^{-1}\right)$ and random coils $\left(1680 \mathrm{~cm}^{-1}\right)$, all of them with a width of $40 \pm 20 \mathrm{~cm}^{-1}$. Additionally, the $1585 \mathrm{~cm}^{-1}$ cytochrome $\mathrm{C}$ peak was also fitted (width $40 \mathrm{~cm}^{-1}$ ). All peaks were allowed a variation in the peak position of $\pm 5 \mathrm{~cm}^{-1}$ except the DC473 fitted peak at $1597 \mathrm{~cm}^{-1}$, that was allowed no variation in position to avoid fitting artefacts in noisy spectra. For the PDT data, the spectra were first filtered by fitting the amplitude to remove spectra where the band was not present. This was done so that the amplitude minus the error was higher than 0.12 for the $1597 \mathrm{~cm}^{-1}$ band and 0.05 for the $2195 \mathrm{~cm}^{-1}$ band. Given the better quality of the spectra from the PDT dataset, the DC473 phenyl peak in this dataset was allowed a shift in the fitting position of $\pm 5 \mathrm{~cm}^{-1}$.

\section{Results}

\section{Detection of DC473 on live single cells}

Fig. 1B shows fluorescent staining of the three cell lines used in this study: SW480, HT29 and SW620. These cell lines 

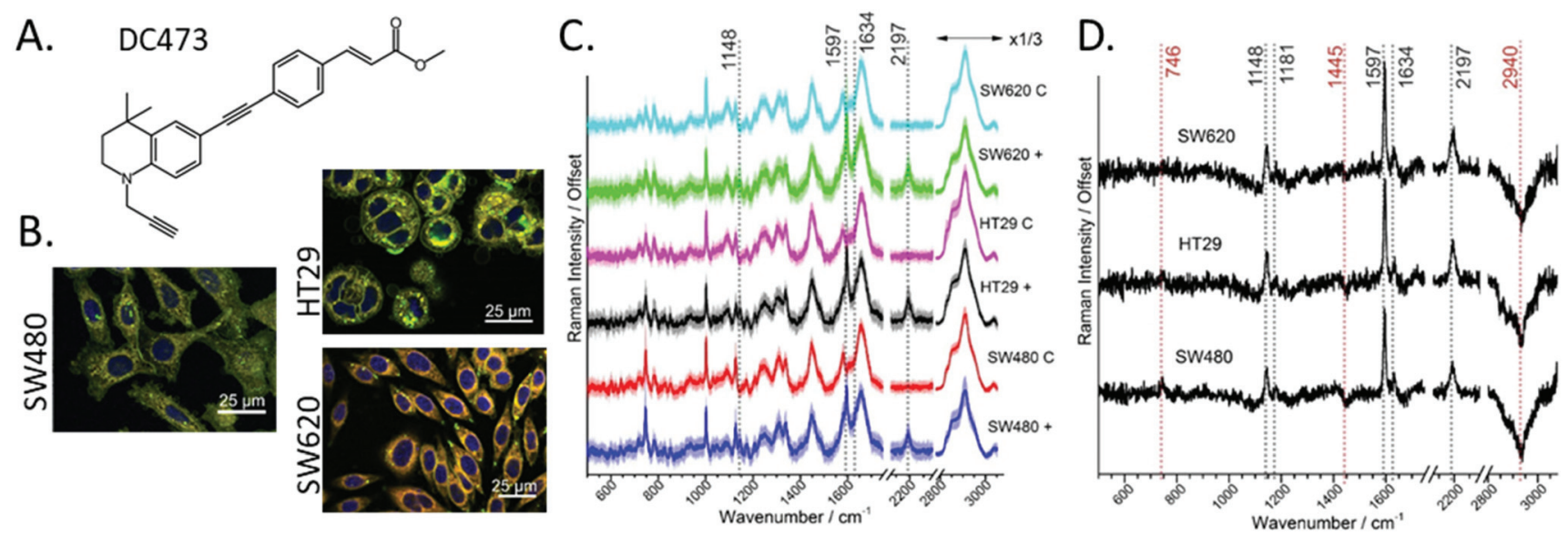

Fig. 1 A. Chemical structure of DC473. B. Confocal fluorescence images of cells for each cell line, where blue is DNA, and green and red are two emission channels for Nile Red: triglycerides (green) and phospholipids (red). C. Average spectra of live single cells of the different cell lines, where + indicates cells following incubation with $4 \mu \mathrm{M}$ of DC473 and C indicates cells with an equivalent volume of DMSO. The area around the curve corresponds to one standard deviation and the main DC473 bands have been indicated. The number of cells in each group is $N \geq 134$. D. Difference spectra of the average between DC473 induced cells and control cells for each of the cell lines. All spectra are normalized to the Amide I peak and have been offset for clarity.

belong to progressive stages of colorectal adenocarcinoma (Duke's stage B, C and C metastasis respectively). Nile red emission is shown in green (triglycerides) and red (phospholipids). ${ }^{70}$ The three cell lines show distinct morphology and the presence of lipid droplets (green), HT29 showed a particularly high quantity of lipid droplets around the nucleus, whereas SW620 showed lipid droplets located near the Poles. SW480 cells showed lipid droplets both around the nucleus and near the Poles. As DC473 is a hydrophobic compound, we expected cells with higher amounts of lipid droplets to have increased uptake of DC473. In a previous study, we showed that DC473 in SW480 cells accumulated in lipid droplets and in the cytosol, presumably on the mitochondrial membrane. ${ }^{25}$

Using a beam expanded laser spot allowed the relatively fast acquisition of high-quality averaged spectra per cell. Fig. 1A shows the chemical structure of DC473. ${ }^{25}$ Fig. 1C shows the average spectra for each of the cell lines and comparing the cells following incubation with DC473, or with DMSO control. All spectra are normalized to the Amide I band. The five strongest peaks from the spectrum of the pure DC473 (see Fig. S1 and Table S1 from the ESI $\dagger$ ) are identified as: $1141 \mathrm{~cm}^{-1}$ (DC473 phenyl), $1597 \mathrm{~cm}^{-1}$ (DC473 phenyl), $1634 \mathrm{~cm}^{-1}$ (DC473 ester) and $2197 \mathrm{~cm}^{-1}$ (DC473 internal alkyne). The terminal alkyne band that would be expected at lower wavenumbers was not observed ${ }^{71}$ which is in agreement with general observations that terminal alkynes often show weaker bands than internal and especially conjugated alkynes. ${ }^{23}$ The average spectra show uptake of the drug for all cell lines, with higher DC473/protein content for HT29 and SW620 cells. DC473 bands were present in all cell lines, where the $1634 \mathrm{~cm}^{-1}$ peak showed as a shoulder on the Amide I band. DMSO was non-detectable in the samples, based on the absence of the $\mathrm{C}-\mathrm{S} \nu_{\mathrm{S}}$ band at $674 \mathrm{~cm}^{-1}$.
Fig. 1D shows the difference spectra indicating the presence of not only the main DC473 bands, but also a reduction in the $\mathrm{CH}$ contribution of both the $1445 \mathrm{~cm}^{-1}$ and the stretching band at $2940 \mathrm{~cm}^{-1}$ following incubation with DC473. This suggests that DC473 could interfere with lipid metabolism and/or regulation. A weak band at $1181 \mathrm{~cm}^{-1}$ was also present in the difference spectra as is associated with the DC473 phenyl peak. We note that incubation with DC473 also correlated with an increase in the $746 \mathrm{~cm}^{-1}$ cytochrome $\mathrm{C}$ band, suggesting that DC473 localised in mitochondria and that it may interfere with organelle function even before PS activation.

\section{DC473 band fitting on single cell spectra}

To compare the DC473 accumulation in the different cell lines, we used Gaussian Lorentzian sum (GLS) single cell peak fitting of the DC473 spectral peaks at $2195 \mathrm{~cm}^{-1}$ and at $1597 \mathrm{~cm}^{-1}$, as detailed in the Methods section. Example fits are shown in Fig. 2B for the $1594 \mathrm{~cm}^{-1}$ band and Fig. 2D for the $2194 \mathrm{~cm}^{-1}$ band. The results from the fitted amplitude are shown in Fig. 2A and C, where the significance between cell types is also indicated (based on $t$-test with unequal variances). All cell lines displayed uptake of DC473, but when comparing the different cell lines (normalizing to the Amide I band), SW620 and HT29 exhibited similar levels of uptake and significantly higher accumulation of DC473 than SW480 cells, likely due to the higher presence of lipid droplets (see Fig. 1B).

For the $2195 \mathrm{~cm}^{-1}$ peak, the position and width of the peak were allowed to vary on a cell by cell basis. Peaks with amplitude lower than 0.15 (normalized to the Amide I band) were excluded. The mean peak amplitude, width and position obtained are shown in Table 1. Cell lines with higher accumulation of DC473 showed broader peaks and a shift towards higher wavenumber. We believe this is related with the local 
A.

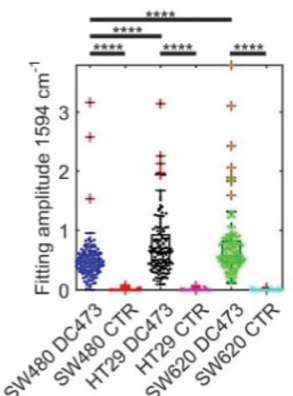

C.

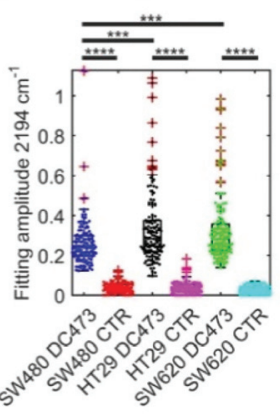

B.

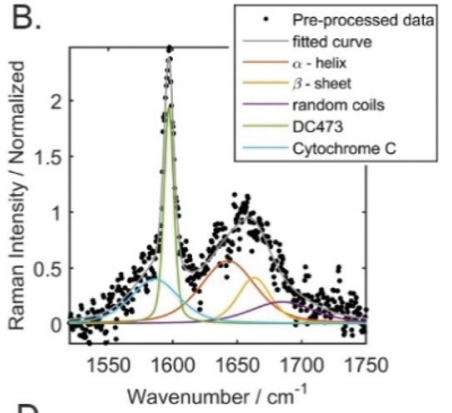

D.

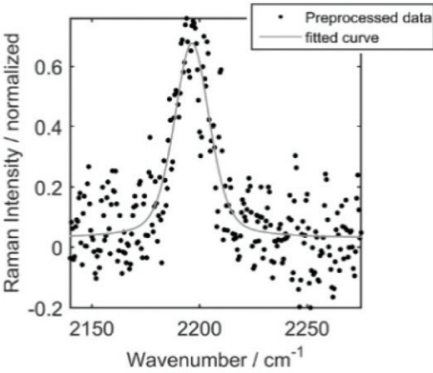

Fig. 2 Comparison of the characteristic DC473 signals in different colorectal single cells following incubation with either DC473, or DMSO as a control. A. Bee swarm and box plots of the amplitudes for the $1594 \mathrm{~cm}^{-1}$ peak fitting. B. Peak fitting for a SW620 cell. C. Bee swarm and box plots of the amplitudes for the $2194 \mathrm{~cm}^{-1}$ peak. D. Peak fitting for the alkyne band associated with the DC473 in the same SW60 cell in (B).

Table 1 Summary of fitting parameters for the alkyne peak at $2197 \mathrm{~cm}^{-1}$ for each of the cell lines. The parameters correspond to the GLS curves \pm SE. The number of cells that had an amplitude $>0.15$ (normalized to Amide I) is shown ( $N$ ), and were used to calculate the remaining parameters

\begin{tabular}{lllll}
\hline & & $\begin{array}{l}\text { Amplitude } \\
\text { (normalized) }\end{array}$ & Position $\left(\mathrm{cm}^{-1}\right)$ & Width $\left(\mathrm{cm}^{-1}\right)$ \\
\hline SW480 & 121 & $0.28 \pm 0.01$ & $2193.9 \pm 0.2$ & $18.8 \pm 0.2$ \\
HT29 & 129 & $0.34 \pm 0.02$ & $2195.2 \pm 0.2$ & $19.7 \pm 0.4$ \\
SW620 & 133 & $0.33 \pm 0.01$ & $2194.8 \pm 0.2$ & $21.0 \pm 0.5$
\end{tabular}

environment where the compound accumulates within the cell, with cell lines with more lipid droplets accumulating more compound in a more hydrophobic environment, with a small shift towards higher wavenumbers.

The $\mathrm{CH}$ stretching band was also analysed at the single cell level, integrating between 2800 and $3080 \mathrm{~cm}^{-1}$ (see Fig. S2 from the ESI $\dagger$ ). Data showed a very significant drop in $\mathrm{CH}_{-}$ stretching bands intensity for all cell lines $\left(p<10^{-4}\right)$ following incubation with DC473.

\section{Principal component analysis}

To further understand the data, PCA was carried out, in which the smoothing of the spectrum was avoided to increase the sensitivity of any band position shifting. Fig. S4A (ESI $\dot{\text { ) }}$ ) shows the first 4 PCA loadings explaining $21.5 \%$ of the total variability, where the main DC473 (black) and cell (red) peaks have

been labelled. Fig. S4B (ESI†) shows the scores for those first 4 PCs. PCA results confirmed the increase in cytochrome $\mathrm{C}$ band intensity and the overall reduction in the $\mathrm{CH}$ stretching intensity following incubation with DC473. This is in line with our previous results showing accumulation in lipid droplets, ${ }^{25}$ indicating that the compound could be partially disrupting lipid accumulation. The positive correlation of cytochrome $\mathrm{C}$ with DC473 suggests that the compound could be interacting with mitochondria.

\section{DC473 activation off cell}

Following our understanding of the accumulation of the compound in these cell lines, further experiments were made to understand the UV activation mechanisms of DC473. Deep UV light was effective in bleaching the fluorescence and led to the loss of the Raman signal of the compound. A $100 \mu \mathrm{M}$ solution of DC473 in DMSO was measured before and after exposure to UV light (340-380 nm, $5.2 \mathrm{~J} \mathrm{~cm}^{-2}$ ). The spectra acquired using the $785 \mathrm{~nm}$ excitation line are shown in Fig. S3 in the ESI, $\uparrow$ normalized to the maximum DMSO band. The exposure to UV light caused a significant drop in the main Raman bands associated with the alkyne and phenyl modes, without the appearance of other secondary bands, indicating that the diphenylacetylene structure of DC473 is degraded due to UV light exposure, and the resultant degradation products are too weak to detect or exhibit Raman bands of comparatively weak intensity. It should be noted that even after prolonged UV exposure, not all the DC473 signal disappeared.

\section{DC473 PDT on live single cells}

To study the effects of the DC473 PS in single cells, the cells were incubated with the compound, then detached from the well plate and resuspended in buffer before analysis. A Raman spectrum in the fingerprint region was acquired before UV treatment. Post UV exposure Raman spectra were acquired every $20 \mathrm{~s}$, for approximately 5 minutes. Two controls without the PS were prepared to separate any potential phototoxicity due to the excitation laser or the UV light used to irradiate the compound. Specifically, cells were either exposed to the same UV dose and the Raman laser or just to the Raman laser excitation. We have already shown that DC473 does not degrade under the $532 \mathrm{~nm}$ laser doses used in this experiment, and thus no control with DC473 and no UV light was undertaken. Measurements were typically made on around 10-11 cells per group, with a total of around 30-35 cells per cell line and in each case 15 spectra per cell.

An example of the data obtained in such an experiment is shown in Fig. 3A. The DC473 bands at 1597 and at $2195 \mathrm{~cm}^{-1}$ showed a strong contribution in the initial spectrum $(t=0)$ which decreased rapidly after PS activation. However, not all the DC473 signal disappeared; a small amount of the compound remained according to the cell spectra. This data agrees with the off-cell data, suggesting that DC473 undergoes a significant structural change following UV activation and consequently, loses its characteristic Raman signal. 

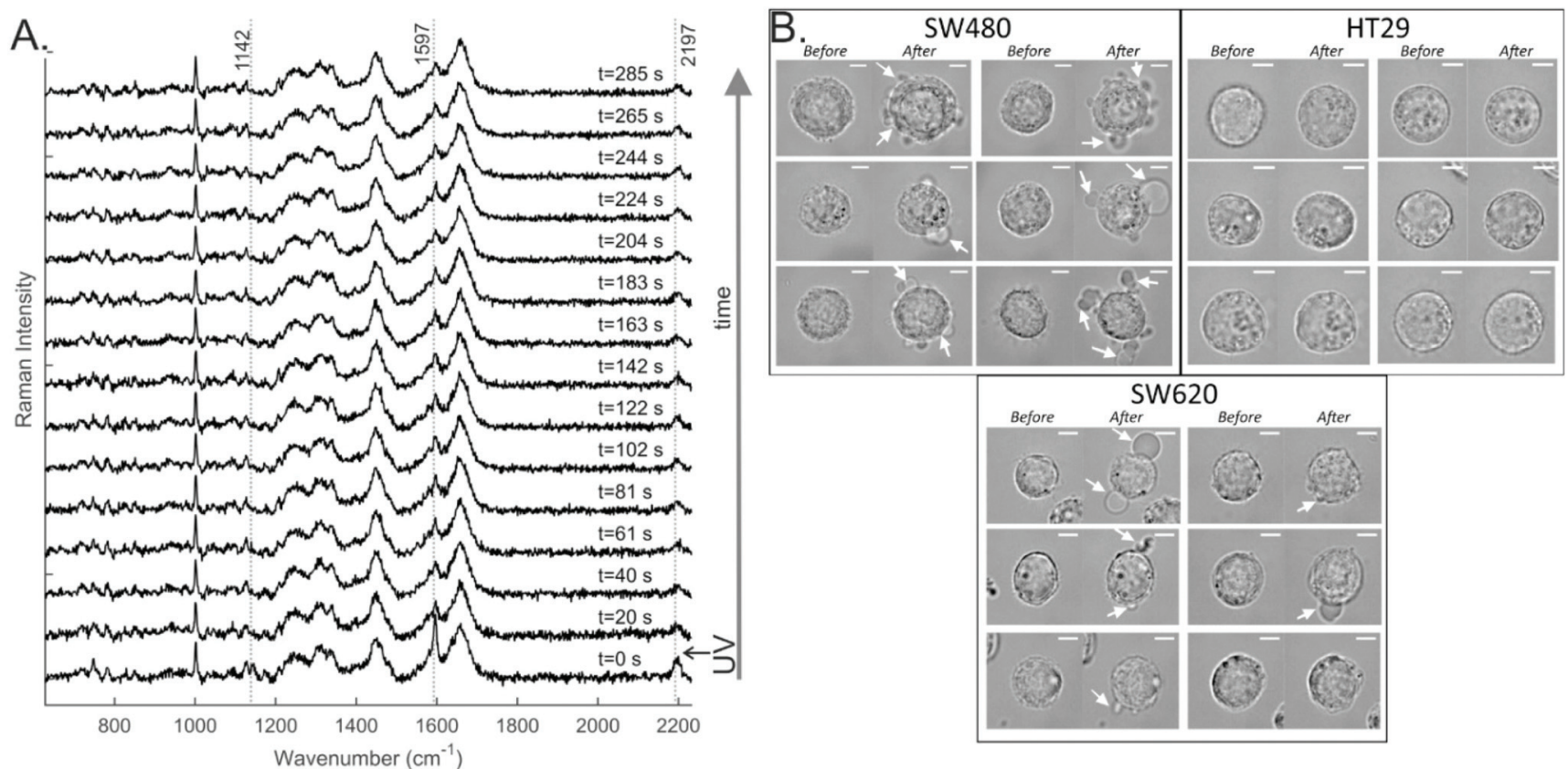

Fig. 3 A. Example of a time-dependent single-cell (SW480) response to the DC473 PDT activation with UV light, where the first spectrum was taken before activation of the photosensitiser, and the remaining spectra were collected post activation. The data was pre-processed as described in Methods. B. Bright field images following incubation with DC473 before and after Raman acquisition following PDT treatment with UV light (approx. 5 minutes). Membrane blebbing was observed, as indicated with white arrows.

Interestingly, changes in the morphology of the treated cells following the Raman acquisition were detectable for some cells from the SW480 and SW620 cell lines, as can be seen in Fig. 3B. Cells would undergo blebbing by the end of the acquisition (indicated with white arrows), a sign of cell death and an expected response of PDT. For the controls, only $3 \%$ of the cells showed morphology changes, with $2 / 30$ cells for the UV control and 0/30 cells for the Raman laser control. From the DC473-treated cells $64 \%$ of SW 480 cells, $0 \%$ of HT 29 cells and $60 \%$ of SW620 cells showed morphological changes following PDT. Surprisingly, although the HT29 and SW620 cells displayed very similar DC473 accumulation they had very different responses to UV activation. However, it must be noted that the absence of morphological changes at the time of measurement does not preclude the cells could undergo apoptosis on longer time scales.

To illustrate the differences in cell responses, the average initial and final spectra for each cell are shown in Fig. 4 (Control, Control + UV and DC473 + UV). These spectra were overlaid for each cell line. For the DC473 + UV samples, the main DC473 bands at 1597 and $2195 \mathrm{~cm}^{-1}$ showed a significant reduction in intensity following PDT, but not always down to undetectable levels (see bands at 1143, 1597, shoulder at 1632 and band at $2195 \mathrm{~cm}^{-1}$ ). In particular, the SW480 and SW620 cells often showed detectable levels of DC473 following UV activation, whilst the HT29 cells showed a drastic reduction of the compound's Raman signal. These results confirmed that the compound was undergoing a chemical modification within the cells. The differences in photodegradation of the compound in different cell lines suggest that the compound

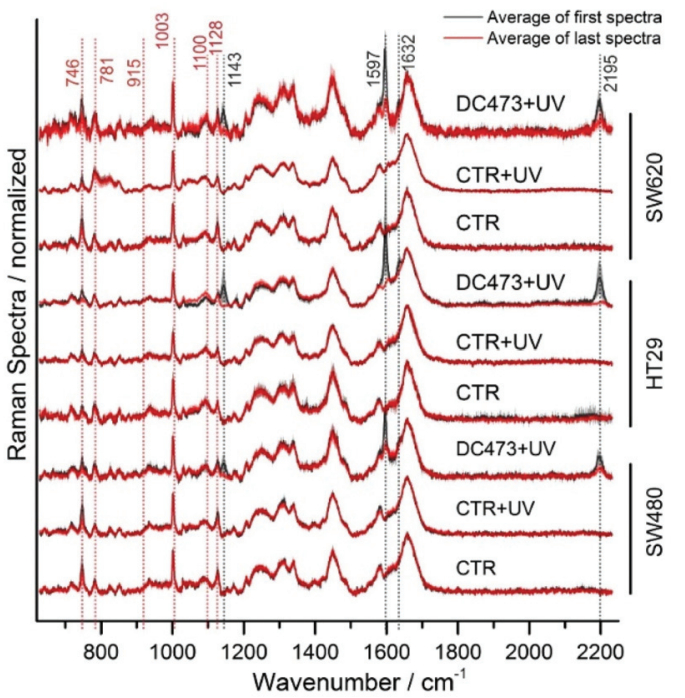

Fig. 4 Average of the data before (black) and after (red) each PDT experiment for each of the samples and cell lines, where CRT = Control, $\mathrm{CRT}+\mathrm{UV}=$ Control exposed to equivalent dose of UV light, DC473 + UV = DC473 sample exposed to UV light. The main bands have been labelled. The area around the curve represents one standard deviation.

may be showing different aggregation states within the cell. Indeed, it has been shown that diphenyacetylene derivatives can form liquid crystalline states in solution and that the highly organised structures formed showed a similar blue shifted absorption band. ${ }^{72}$ As the compound accumulates both in lipid droplets and in the cytosol, the coexistence of 
these two states is expected, with the result that these aggregates would be less susceptible to photoactivation due to their absorption band being shifted. When comparing different cell lines, the differences observed in the cell's morphological changes post treatment can also be linked to the degradation of the compound, showing that cell lines with remaining DC473 signal also showed more blebbing, post-treatment. To understand the observed morphology changes, it must be noted that the cells may have different resistances to oxidative stress. SW480 and SW620 cells show KRAS (G12V) mutation, and HT29 cells show BRAF (V600E) mutation, ${ }^{73}$ however, the relation between these mutations and the redox state is not well understood. ${ }^{74}$ Furthermore, previous reports showed that lipid droplets can have a protective effect against reactive oxygen species, ${ }^{75}$ which could explain why HT29 cells showed higher accumulation but no response to the activation of DC473, compared to SW480 and SW620 cells, that showed fewer lipid droplets. Additionally, the location of the compound in each cell line might be different and could have a strong impact in its toxicity following activation. Regarding the controls, whilst the signal remained largely unchanged, the cytochrome C bands were observed to decrease (746 and $1128 \mathrm{~cm}^{-1}$ ) in all treatments indicating some phototoxicity due to both the PDT, the Raman acquisition laser and UV light dose.

When calculating the difference between the average spectra, the main effects of the PDT became evident (Fig. S5, ESI $\dagger$ ). Results showed a drop in all DC473 peaks and in cell bands at 1443, 1181, 1597, 1632 and $2195 \mathrm{~cm}^{-1}$. All datasets showed negative peaks corresponding to the cytochrome C progression (746, 1128, 1310 and 1585), indicating phototoxicity of the excitation $532 \mathrm{~nm}$ laser light and of the UV light, with also DNA signal loss associated with degradation in some of the samples observed at $781 \mathrm{~cm}^{-1}$. Additional changes were observed in the band at $1338 \mathrm{~cm}^{-1}$, which has mixed contributions from proteins and nucleic acids, in the lipid band at $1445 \mathrm{~cm}^{-1}$ and on the side of the Amide I at $1674 \mathrm{~cm}^{-1}$.

\section{PCA analysis of DC473 PDT on live single cells}

To explore these changes further, PCA was performed. Fig. 5A shows the loadings for the first 5 coefficients. Fig. S5 (ESI $\dagger$ ) shows the variation of the average scores for each of the conditions and cell lines with time, where the intensity of all traces was aligned to 0 , for $t=0$ to better see the trends with time. The principal component PC2 was mainly associated with contributions from DC473, whilst PC3 was associated with cytochrome $\mathrm{C}$ and PC4 with the unassigned bands at 1314, 1338, 1362 and $1390 \mathrm{~cm}^{-1}$ which showed a negative trend over time, being potential biomarkers of the cell response to DC473 activation. PCA was unable to identify any clear Raman marker for the cell blebbing (morphology changes) observed after PDT, and only showed a strong reduction on PC3 and 4 on all treated cells, in line with the amount of DC473 that the cells accumulated. A 2D plot of the single-cell score traces for PC2 and PC4 is shown in Fig. 5B as a summary of the PCA findings. The initial point for each cell was shown with a marker, and the subsequent points were shown as a line trace. The HT29 control group did not show overall changes with time, whereas SW480 and SW620 cells showed a drop in the PC4 score over time suggesting these cells were more sensitive to the phototoxicity of the $532 \mathrm{~nm}$ excitation laser and the UV light. PDT samples showed a dramatic reduction in both coefficients immediately after the UV dose.

\section{Single-cell band fitting of DC473 bands following PDT}

To better characterize the changes that DC473 underwent following UV light activation, both the 1592 and the $2195 \mathrm{~cm}^{-1}$

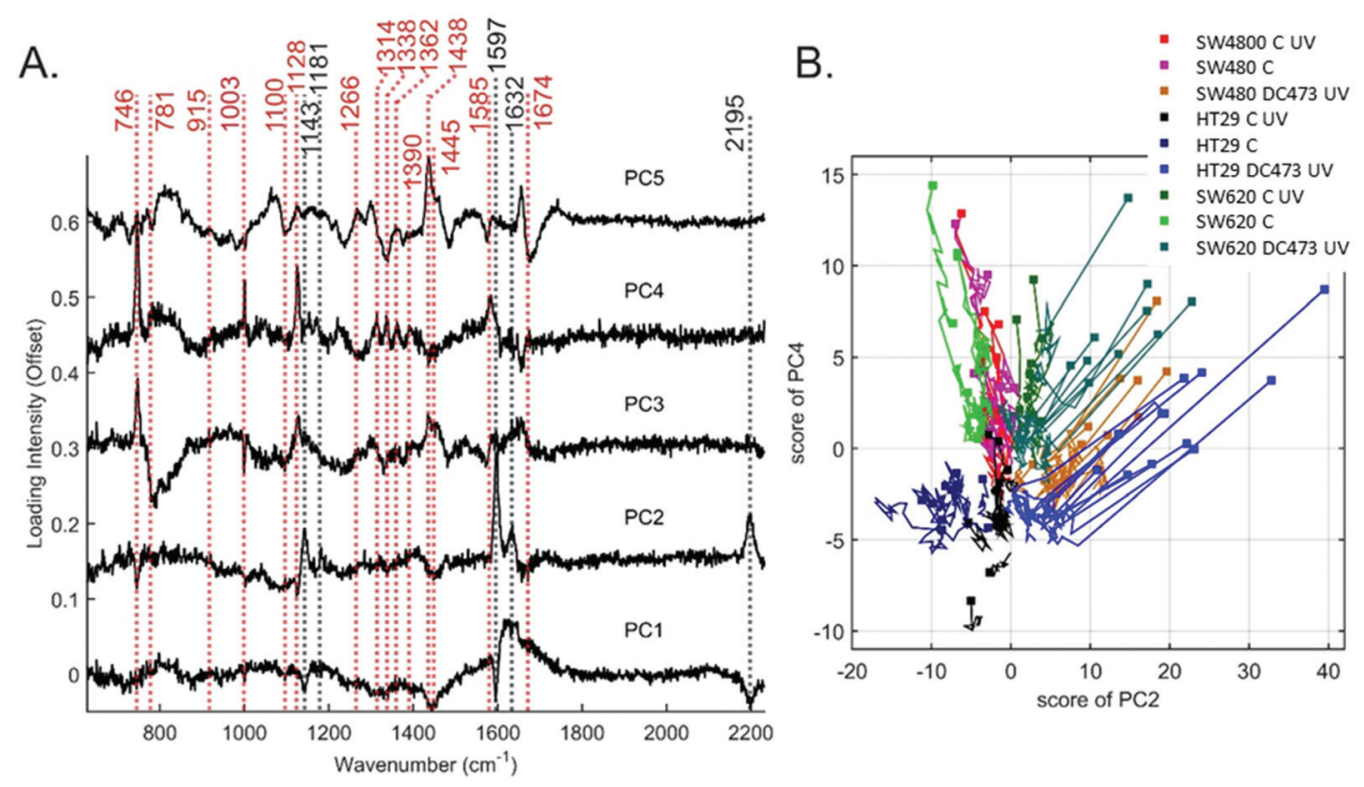

Fig. 5 A. PCA loadings for the first 5 PCs for the PDT data. The main cell peaks (red) and DC473 peaks (black) have been labelled. B. PCA traces for PC4 and PC2 for the PDT data, where the first time point is shown as a square, and remaining observations in that cell are shown as a trace. 

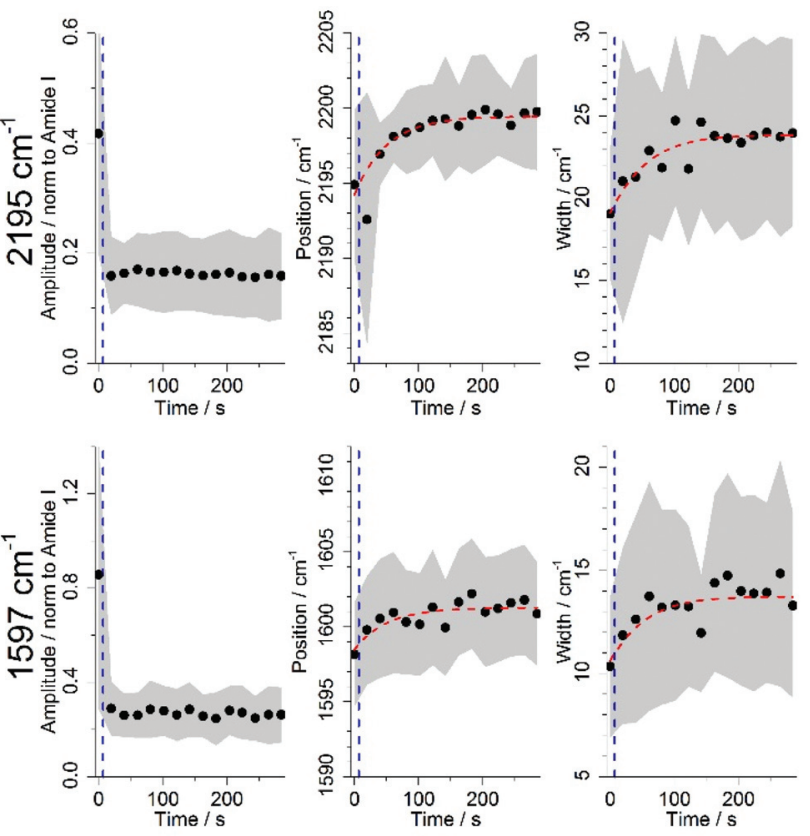

Fig. 6 Average time dependence of the fitting parameters for the 2195 and $1597 \mathrm{~cm}^{-1}$ band, for all cell lines incubated with DC473 and treated with UV light after amplitude filtering, where the area behind the curve corresponds to one standard deviation. The point at which the UV dose was applied has been represented with a vertical blue dashed line. Red dashed lines show the sigmoid fittings done to the average data.

bands were fitted to Gaussian Lorentzian Sum (GLS) functions. Fig. 6 shows the average results for all cells after the amplitude filtering on both DC473 bands, where the area around the curves corresponds to one standard deviation, and each data point was an average of at least 20 fittings. As a lot of cells showed too low band intensity for fitting, all cell lines were analysed together. Results showed a sharp drop in the amplitude of both bands caused by the UV irradiation, followed by a plateau. However, the position and widths of the bands showed a slower time-dependent trend post-UV exposure. The four curves were fitted to a sigmoid function over time $(y=$ $a /(a+b \cdot \exp (-\tau \cdot t)))$, where $a$ is the asymptote of the sigmoid $\left(\mathrm{cm}^{-1}\right), b$ is the ratio between the asymptotic shift and the initial value (adimensional) and $\tau\left(\mathrm{s}^{-1}\right)$ is the inverse of the lifetime. The values for the parameters of the fitted curves are shown on Table $2( \pm \mathrm{SE})$.

The position of the $2195 \mathrm{~cm}^{-1}$ band shifted towards higher wavenumbers, peaking at $2199.5 \mathrm{~cm}^{-1}$, for a total shift of $\sim 4.8 \mathrm{~cm}^{-1}$. The $1597 \mathrm{~cm}^{-1}$ band had a slightly smaller shift to $1601.3 \mathrm{~cm}^{-1}\left(\sim 3.6 \mathrm{~cm}^{-1}\right)$. In terms of the GLS width, the alkyne band showed an increase in width of $\sim 4.9 \mathrm{~cm}^{-1}$ whilst the sharper phenyl band showed a modest increase in width of around $\sim 3.0 \mathrm{~cm}^{-1}$. Both bands showed a similar $\tau$ constants of $0.02 \mathrm{~s}^{-1}$. The inverse of $\tau$, or the lifetime of these events, is of approximately $50 \mathrm{~s}$. It has previously been reported with phenylacetylene that increased order in a liquid matrix leaded to narrower bands and shifted maximum intensity to lower wavenumbers. ${ }^{76}$ We believe that PDT causes the DC473 aggre-
Table 2 Time dependence sigmoid fittings of the DC473 average band GLS fitting parameters: band position and width $\left(\mathrm{cm}^{-1}\right)$. The corresponding fittings are shown in Fig. 6

\begin{tabular}{llll}
\hline \multirow{4}{*}{ Band } & \multicolumn{3}{l}{ Position $/ \mathrm{cm}^{-1}$} \\
\cline { 2 - 4 } & $a / \mathrm{cm}^{-1}$ & $b$ & $\tau / \mathrm{s}^{-1}$ \\
\hline 2195 & $2199.5 \pm 0.2$ & $0.0024 \pm 0.0003$ & $0.020 \pm 0.004$ \\
1597 & $1601.3 \pm 0.3$ & $0.0018 \pm 0.0004$ & $0.02 \pm 0.01$ \\
\hline & Width $/ \mathrm{cm}^{-1}$ & \\
\cline { 2 - 4 } & $a / \mathrm{cm}^{-1}$ & $b$ & $\tau / \mathrm{s}^{-1}$ \\
\hline 2195 & $23.9 \pm 0.4$ & $0.25 \pm 0.04$ & $0.021 \pm 0.007$ \\
1597 & $13.7 \pm 0.4$ & $0.29 \pm 0.08$ & $0.02 \pm 0.01$
\end{tabular}

gates, which are resistant to photodegradation, ${ }^{72}$ to disaggregate, causing the Raman band to widen and to shift to higher wavenumbers. Additionally, the biological environment of the drug has a very important role in this process, by either stabilizing or destabilizing these aggregates, and providing substrates for reaction with the compound.

The two most susceptible cell lines to PDT, based on morphological changes, showed residual DC473 post-PDT, whereas the HT29 cells showed an absence of any remaining DC473 post-PDT, in most observations. In our previous publication we observed that DC473 localized in both lipid droplets and in the cytosol. ${ }^{25}$ We believe that aggregates could be forming with a shifted absorption band compared to the free compound, and thus a lower response to the UV light. Upon PDT, the aggregates would be disrupted, showing a widening and shift of the Raman band to higher wavenumbers. The presence of aggregates anti-correlates with the morphological response of the cells, however, more cell lines need to be studied to confirm this result. Additionally, the inherent concentration of potential substrates for reaction with the activated compound in each cell line is an important factor to explain their different response to PDT. Further experiments are needed to confirm the dual behaviour of the compound and to fully understand the cell toxicity mechanism. Nevertheless, Raman on live cells proves to be a powerful tool to track the timedynamics to drug responses at the single-cell level.

\section{Conclusions}

Our experiments indicate that DC473 can be readily taken up by different CRC cell lines. Raman spectroscopy results indicated that accumulation mainly occurs in lipid droplets and the cytosol, and was significantly higher for more advanced adenocarcinoma HT29 and SW620 cells compared to SW480 cells. This is likely linked to their higher lipid droplet content. However, when activating the compound with UV light, SW480 and SW620 showed clear morphological changes in response to the drug, whereas HT29 cells showed no change. The DC473 Raman signal reduced upon activation, however, some 
inactivated compound remained for SW480 and SW620 cells, whilst there was minimal compound left in HT29 cells. It is known that diphenylacetylenes can form highly organised aggregates with blue shifted absorption bands, ${ }^{72}$ which would make them less susceptible to the PDT UV light. Multivariate analysis identified coefficients that showed time-dependent changes in multiple cell peaks over time after DC473 activation, including cytochrome C bands. The main two DC473 peaks at 1597 and $2195 \mathrm{~cm}^{-1}$ showed a significant drop in intensity after irradiation and additional shift to higher wavenumbers and increase in peak width over time at the single cell level. These changes happened with a lifetime of approximately $50 \mathrm{~s}$. These effects possibly indicate a change in the aggregation state of the compound, with disruption of the aggregates causing the widening and shift of the bands to higher wavenumbers. ${ }^{76}$ Our results indicate that the compound could be suitable for CRC PDT, with higher accumulation on lipid droplet rich cancer stages. However, its effects in lipid metabolism need further understanding and some cells could be resistant to this treatment, as the data for HT29 seems to indicate. The results showed limited phototoxicity due to the UV and Raman excitation light sources and that this may also be different on different cell lines. Finally, the results also highlight heterogeneity both in the accumulation and response to PDT both between and within cell lines, underlining the importance of single cell studies. Further research needs to be done to understand the biological mechanism behind phototoxicity of the compound in these cell lines. These findings confirm the feasibility of doing live single cell drug studies using RS, with sensitivity to cell viability and chemical and environmental changes in the drug over time.

\section{Conflicts of interest}

AW and CAA are shareholders and directors of LightOx Ltd, a company licensed to pursue commercial applications for the novel compound described in this manuscript.

\section{Acknowledgements}

JGP thanks EPSRC for the PhD studentship, the University of Leeds Alumni for financial support and JSPS. DC thanks EPSRC, BBSRC and High Force Research Ltd for funding. SDE acknowledges the following funders; MRC (MR/M009084/1) and EPSRC (EP/P023266/1). SDE is supported by the National Institute for Health Research (NIHR) infrastructure at Leeds. The views expressed are those of the author(s) and not necessarily those of the NHS, the NIHR or the Department of Health. We acknowledge Catriona Marshal (SJUH) for providing the cell lines and STR validation. We would like to thank Jon Girkin and Valerie Affleck for their input and advice. The data used in the figures of this paper will be available at https:/doi. org/10.5518/702.

\section{Notes and references}

1 M. K. Hammoud, H. K. Yosef, T. Lechtonen, K. Aljakouch, M. Schuler, W. Alsaidi, I. Daho, A. Maghnouj, S. Hahn, S. F. El-Mashtoly and K. Gerwert, Sci. Rep., 2018, 8, 15278.

2 I. W. Schie and T. Huser, Appl. Spectrosc., 2013, 67, 813828.

3 C. Krafft, M. Schmitt, I. W. Schie, D. Cialla-May, C. Matthäus, T. Bocklitz and J. Popp, Angew. Chem., Int. Ed., 2017, 56, 4392-4430.

4 N. K. Das, Y. Dai, P. Liu, C. Hu, L. Tong, X. Chen and Z. J. Smith, Sensors, 2017, 17, 1592.

5 R. Smith, K. L. Wright and L. Ashton, Analyst, 2016, 141, 3590-3600.

6 K. Kong, C. Kendall, N. Stone and I. Notingher, Adv. Drug Delivery Rev., 2015, 89, 121-134.

7 I. Notingher and L. L. Hench, Expert Rev. Med. Devices, 2006, 3, 215-234.

8 I. W. Schie, J. Rüger, A. S. Mondol, A. Ramoji, U. Neugebauer, C. Krafft and J. Popp, Anal. Chem., 2018, 90, 2023-2030.

9 J. Phillip Kennedy, P. Jeffrey Conn and C. W. Lindsley, Bioorg. Med. Chem. Lett., 2009, 19, 3204-3208.

10 M. Diem, A. Mazur, K. Lenau, J. Schubert, B. Bird, M. Miljković, C. Krafft and J. Popp, J. Biophotonics, 2013, 6, 855-886.

11 H. J. Byrne, M. Baranska, G. J. Puppels, N. Stone, B. Wood, K. M. Gough, P. Lasch, P. Heraud, J. Sulé-Suso and G. D. Sockalingum, Analyst, 2015, 140, 2066-2073.

12 G. P. S. Smith, C. M. McGoverin, S. J. Fraser and K. C. Gordon, Adv. Drug Delivery Rev., 2015, 89, 21-41.

13 B. Kann, H. L. Offerhaus, M. Windbergs and C. Otto, Adv. Drug Delivery Rev., 2015, 89, 71-90.

14 C. Matthäus, A. Kale, T. Chernenko, V. Torchilin and M. Diem, Mol. Pharm., 2008, 5, 287-293.

15 F. Hu, L. Wei, C. Zheng, Y. Shen and W. Min, Analyst, 2014, 139, 2312-2317.

16 S. Hong, L. Lin, M. Xiao and X. Chen, Curr. Opin. Chem. Biol., 2015, 24, 91-96.

17 W. J. Tipping, M. Lee, A. Serrels, V. G. Brunton and A. N. Hulme, Chem. Soc. Rev., 2016, 45, 2075-2089.

18 H. Yamakoshi, A. F. Palonpon, K. Dodo, J. Ando, S. Kawata, K. Fujita and M. Sodeoka, Chem. Commun., 2014, 50, 13411343.

19 M. Okada, N. I. Smith, A. F. Palonpon, H. Endo, S. Kawata, M. Sodeoka and K. Fujita, Proc. Natl. Acad. Sci. U. S. A., 2012, 109, 28-32.

20 J. Gala de Pablo, F. J. Armistead, S. A. Peyman, D. Bonthron, M. Lones, S. Smith and S. D. Evans, J. Raman Spectrosc., 2018, 49, 1323-1332.

21 S. Hong, T. Chen, Y. Zhu, A. Li, Y. Huang and X. Chen, Angew. Chem., Int. Ed., 2014, 53, 5827-5831.

22 H. Yamakoshi, K. Dodo, M. Okada, J. Ando, A. Palonpon, K. Fujita, S. Kawata and M. Sodeoka, J. Am. Chem. Soc., 2011, 133, 6102-6105. 
23 H. Yamakoshi, K. Dodo, A. Palonpon, J. Ando, K. Fujita, S. Kawata and M. Sodeoka, J. Am. Chem. Soc., 2012, 134, 20681-20689.

24 F. Hu, C. Zeng, R. Long, Y. Miao, L. Wei, Q. Xu and W. Min, Nat. Methods, 2018, 15, 194-200.

25 J. Gala de Pablo, D. R. Chisholm, A. Steffen, A. K. Nelson, C. Mahler, T. B. Marder, S. A. Peyman, J. M. Girkin, C. A. Ambler, A. Whiting and S. D. Evans, Analyst, 2018, 143, 6113-6120.

26 A. F. Palonpon, M. Sodeoka and K. Fujita, Curr. Opin. Chem. Biol., 2013, 17, 708-715.

27 H. Mikami, C. Lei, N. Nitta, T. Sugimura, T. Ito, Y. Ozeki and K. Goda, Chem, 2018, 4, 2278-2300.

28 M. Li, J. Xu, M. Romero-Gonzalez, S. A. Banwart and W. E. Huang, Curr. Opin. Biotechnol., 2012, 23, 56-63.

29 C. Krafft and J. Popp, Anal. Bioanal. Chem., 2015, 407, 699-717.

30 H. K. Yosef, L. Mavarani, A. Maghnouj, S. Hahn, S. F. ElMashtoly and K. Gerwert, Anal. Bioanal. Chem., 2015, 407, 8321-8331.

31 H. K. Yosef, T. Frick, M. K. Hammoud, A. Maghnouj, S. Hahn, K. Gerwert and S. F. El-Mashtoly, Analyst, 2018, 143, 6069-6078.

32 S. F. El-Mashtoly, H. K. Yosef, D. Petersen, L. Mavarani, A. Maghnouj, S. Hahn, C. Kötting and K. Gerwert, Anal. Chem., 2015, 87, 7297-7304.

33 D. Fu, J. Zhou, W. S. Zhu, P. W. Manley, Y. K. Wang, T. Hood, A. Wylie and X. S. Xie, Nat. Chem., 2014, 6, 614622.

34 S. F. El-Mashtoly, D. Petersen, H. K. Yosef, A. Mosig, A. Reinacher-Schick, C. Kötting and K. Gerwert, Analyst, 2014, 139, 1155.

35 K. Aljakouch, T. Lechtonen, H. K. Yosef, M. K. Hammoud, W. Alsaidi, C. Kötting, C. Mügge, R. Kourist, S. F. ElMashtoly and K. Gerwert, Angew. Chem., Int. Ed., 2018, 57, 7250-7254.

36 H. Nawaz, F. Bonnier, P. Knief, O. Howe, F. M. Lyng, A. D. Meade and H. J. Byrne, Analyst, 2010, 135, 3070.

37 H. Nawaz, F. Bonnier, A. D. Meade, F. M. Lyng and H. J. Byrne, Analyst, 2011, 136, 2450.

38 Z. Farhane, F. Bonnier and H. J. Byrne, Anal. Bioanal. Chem., 2017, 409, 1333-1346.

39 Z. Farhane, F. Bonnier, O. Howe, A. Casey and H. J. Byrne, J. Biophotonics, 2018, 11, 1-14.

40 C. Matthäus, T. Chernenko, C. Stiebing, L. Quintero, M. Miljkovi, L. Milane, A. Kale, M. Amiji, S. Lorkowski and V. Torchilin, Confocal Raman Microscopy, Springer International Publishing, Cham, 2018, vol. 66.

41 A. A. van Apeldoorn, H.-J. van Manen, J. M. Bezemer, J. D. de Bruijn, C. A. van Blitterswijk and C. Otto, J. Am. Chem. Soc., 2004, 126, 13226-13227.

42 P. Xu, E. Gullotti, L. Tong, C. B. Highley, D. R. Errabelli, T. Hasan, J.-X. Cheng, D. S. Kohane and Y. Yeo, Mol. Pharm., 2009, 6, 190-201.

43 G. Romero, I. Estrela-Lopis, J. Zhou, E. Rojas, A. Franco, C. S. Espinel, A. G. Fernandez, C. Gao, E. Donath and S. E. Moya, Biomacromolecules, 2010, 11, 2993-2999.
44 T. Chernenko, C. Matthäus, L. Milane, L. Quintero, M. Amiji and M. Diem, ACS Nano, 2009, 3, 3552-3559.

45 T. Chernenko, F. Buyukozturk, M. Miljkovic, R. Carrier, M. Diem and M. Amiji, Drug Delivery Transl. Res., 2013, 3, 575-586.

46 F. Bray, J. Ferlay, I. Soerjomataram, R. L. Siegel, L. A. Torre and A. Jemal, CA-Cancer J. Clin., 2018, 68, 394-424.

47 C. C. Applegate and M. A. Lane, World J. Gastrointest. Oncol., 2015, 7, 184.

48 R. Abdel-Samad, P. Aouad, H. Gali-Muhtasib, Z. Sweidan, R. Hmadi, H. Kadara, E. L. D’Andrea, A. Fucci, C. Pisano and N. Darwiche, Am. J. Cancer Res., 2018, 8, 39-55.

49 A. Kawczyk-Krupka, A. M. Bugaj, W. Latos, K. Zaremba, K. Wawrzyniec, M. Kucharzewski and A. Sieroń, Photodiagn. Photodyn. Ther., 2016, 13, 158-174.

50 A. Whiting and C. A. Ambler, Synthetic retinoid (in cell killing) UK Patent Application No. GB16137127, 2016.

51 D. R. Chisholm, R. Lamb, T. Pallett, V. Affleck, C. Holden, J. Marrison, P. O’Toole, P. D. Ashton, K. Newling, A. Steffen, A. K. Nelson, C. Mahler, R. Valentine, T. S. Blacker, A. J. Bain, J. Girkin, T. B. Marder, A. Whiting and C. A. Ambler, Chem. Sci., 2019, 10, 4673-4683.

52 T. L. Freeman, S. E. Cope, M. R. Stringer, J. E. CruseSawyer, D. N. Batchelder and S. B. Brown, J. Raman Spectrosc., 1997, 28, 641-643.

53 H. Abramczyk, B. Brozek-Pluska, J. Surmacki, J. Musial and R. Kordek, Analyst, 2014, 139, 5547-5559.

54 H. Abramczyk, B. Brozek-Pluska, M. Tondusson and E. Freysz, J. Phys. Chem. C, 2013, 117, 4999-5013.

55 B. Brozek-Pluska and M. Kopec, Spectrochim. Acta, Part A, 2016, 169, 182-191.

56 B. Brozek-Pluska, A. Jarota, J. Jablonska-Gajewicz, R. Kordek, W. Czajkowski and H. Abramczyk, Technol. Cancer Res. Treat., 2012, 11, 317-331.

57 T. Bhattacharjee, L. C. Fontana, L. Raniero and J. FerreiraStrixino, J. Raman Spectrosc., 2018, 49, 786-791.

58 G. Lajos, D. Jancura, P. Miskovsky, J. V. García-Ramos and S. Sanchez-Cortes, J. Phys. Chem. C, 2009, 113, 71477154.

59 Y. K. Kim, H. K. Na, S. Kim, H. Jang, S. J. Chang and D. H. Min, Small, 2015, 11, 2527-2535.

60 T. Nagy-Simon, M. Potara, A. M. Craciun, E. Licarete and S. Astilean, J. Colloid Interface Sci., 2018, 517, 239-250.

61 N. Nwahara, O. J. Achadu and T. Nyokong, J. Photochem. Photobiol., A, 2018, 359, 131-144.

62 T. Simon, M. Potara, A. M. Gabudean, E. Licarete, M. Banciu and S. Astilean, ACS Appl. Mater. Interfaces, 2015, 7, 16191-16201.

63 L. Zhao, T. H. Kim, H. W. Kim, J. C. Ahn and S. Y. Kim, Acta Biomater., 2015, 20, 155-164.

64 Z. Zhou, S. Peng, M. Sui, S. Chen, L. Huang, H. Xu and T. Jiang, Colloids Surf., B, 2018, 161, 394-402.

65 A. M. Fales, H. Yuan and T. Vo-Dinh, Mol. Pharm., 2013, 10, 2291-2298.

66 A. M. Fales, B. M. Crawford and T. Vo-Dinh, ACS Omega, 2016, 1, 730-735. 
67 A. Farhadi, Á. Roxin, B. C. Wilson and G. Zheng, Theranostics, 2015, 5, 469-476.

68 M. Koch, C. Suhr, B. Roth and M. Meinhardt-Wollweber, J. Raman Spectrosc., 2017, 48, 336-342.

69 V. Jain, M. C. Biesinger and M. R. Linford, Appl. Surf. Sci., 2018, 447, 548-553.

70 N. Ridgway and R. McLeod, Biochemistry of Lipids, Lipoproteins and Membranes, Elsevier Science, 6th edn, 2015.

71 Y. Chen, J. Q. Ren, X. G. Zhang, D. Y. Wu, A. G. Shen and J. M. Hu, Anal. Chem., 2016, 88, 6115-6119.
72 J. Tong, Y. J. Wang, Z. Wang, J. Z. Sun and B. Z. Tang, J. Phys. Chem. C, 2015, 119, 21875-21881.

73 D. Ahmed, P. W. Eide, I. A. Eilertsen, S. A. Danielsen, M. Eknæs, M. Hektoen, G. E. Lind and R. A. Lothe, Oncogenesis, 2013, 2, e71.

74 J. K. M. Lim and G. Leprivier, Cell Death Dis., 2019, 10, 955.

75 P. Shyu, X. F. A. Wong, K. Crasta and G. Thibault, Biosci. Rep., 2018, 38, 1-20.

76 H. Abramczyk and G. Waliszewska, J. Phys. Chem. A, 1998, 102, 7765-7771. 